
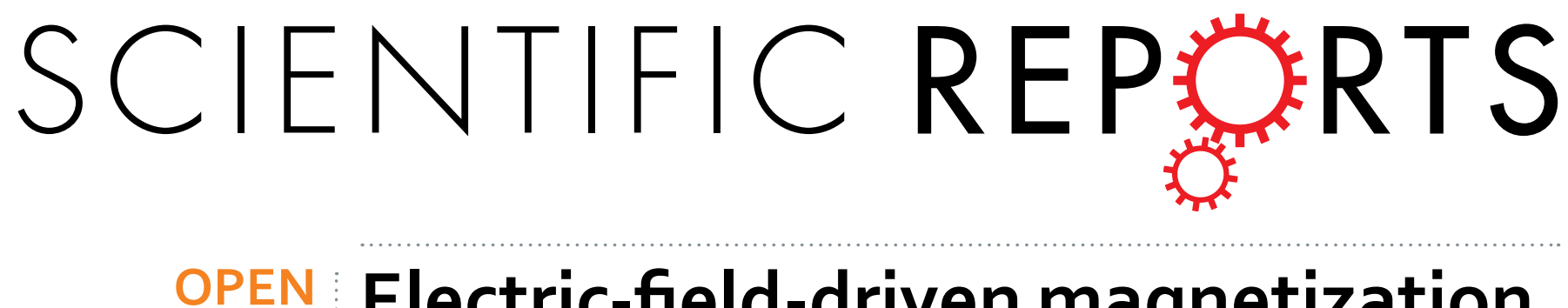

\title{
Electric-field-driven magnetization switching and nonlinear magnetoelasticity in $\mathrm{Au} / \mathrm{FeCo} / \mathrm{MgO}$ heterostructures
}

Received: 14 April 2016

Accepted: 24 June 2016

Published: 18 July 2016

\author{
P. V. Ong ${ }^{1,+}{ }^{+}$Nicholas Kioussis $^{1}$, P. Khalili Amiri ${ }^{2,3}$ \& K. L. Wang ${ }^{2}$
}

Voltage-induced switching of magnetization, as opposed to current-driven spin transfer torque switching, can lead to a new paradigm enabling ultralow-power and high density instant-on nonvolatile magnetoelectric random access memory (MeRAM). To date, however, a major bottleneck in optimizing the performance of MeRAM devices is the low voltage-controlled magnetic anisotropy (VCMA) efficiency (change of interfacial magnetic anisotropy energy per unit electric field) leading in turn to high switching energy and write voltage. In this work, employing ab initio electronic structure calculations, we show that epitaxial strain, which is ubiquitous in MeRAM heterostructures, gives rise to a rich variety of VCMA behavior with giant VCMA coefficient $\left(\sim 1800 \mathrm{fJ} \mathrm{V}^{-1} \mathrm{~m}^{-1}\right)$ in $\mathrm{Au} / \mathrm{FeCo} / \mathrm{MgO}$ junction. The heterostructure also exhibits a strain-induced spin-reorientation induced by a nonlinear magnetoelastic coupling. The results demonstrate that the VCMA behavior is universal and robust in magnetic junctions with heavy metal caps across the $5 d$ transition metals and that an electric-fielddriven magnetic switching at low voltage is achievable by design. These findings open interesting prospects for exploiting strain engineering to harvest higher efficiency VCMA for the next generation MeRAM devices.

Electric field (E-field) control of the magnetization vector via the magnetoelectric effect has sparked an explosion of technological and research interest due to its potential application in ultra-low power, highly-scalable, and non-volatile spin-based random access memory or MeRAM ${ }^{1-4}$. The realization of MeRAM is based on the voltage-controlled magnetic anisotropy (VCMA) of heavy-metal/ferromagnet/insulator (HM/FM/I) nano-junctions, where the non-magnetic $\mathrm{HM}$ contact electrode (Ta, $\mathrm{Pd}, \mathrm{Pt}, \mathrm{Au}$ ) has strong spin-orbit coupling (SOC). In the linear regime, the VCMA is proportional to the E-field in the insulator, VCMA $=\beta E_{I}=\beta E_{\text {ext }} / \varepsilon_{\perp}$, where $\beta$ is the VCMA coefficient, $E_{\text {ext }}$ is the external E-field, and $\varepsilon_{\perp}$ is the out-of-plane component of the relative dielectric constant tensor of the insulator. The challenge for achieving a switching energy per bit below that in complementary metal oxide semiconductor $(\sim 1 \mathrm{fJ})$ and a write voltage below $1 \mathrm{~V}$ requires large perpendicular magnetic anisotropy (PMA) $)^{1,5}$ and a VCMA coefficient higher than $\sim 200 \mathrm{fJ} \mathrm{V}^{-1} \mathrm{~m}^{-16}$.

The VCMA of HM/FM/I junctions depends on the HM cap, the particular FM material or its alloys and exhibits a wide range of behavior ranging from linear to nonmonotonic $\vee$-shape or inverse- $\vee$-shape $(\wedge)$ E-field dependence with asymmetric $\beta$ 's. A linear VCMA was observed in $\mathrm{Ta} / \mathrm{Co}_{40} \mathrm{Fe}_{40} \mathrm{~B}_{20} / \mathrm{MgO}^{7}$ and in $\mathrm{Pd} / \mathrm{FePd} / \mathrm{MgO}^{8}$ tunnel junctions with $\beta$ of -33 and $+600 \mathrm{fJV}^{-1} \mathrm{~m}^{-1}$, respectively, where the convention of positive E-field corresponds to electron accumulation at the $\mathrm{FM} / \mathrm{I}$ interface. On the other hand, recent experiments on $\mathrm{V} / \mathrm{Fe} / \mathrm{MgO}$ revealed an asymmetric $\wedge$-shape VCMA with giant $\beta$ values of $1150 \mathrm{fJ} \mathrm{V}^{-1} \mathrm{~m}^{-19}$, while a $\vee$-shape VCMA was observed in double-barrier $\mathrm{MgO} / \mathrm{FeB} / \mathrm{MgO} / \mathrm{Fe}$ junctions with $\beta=100 \mathrm{fJ} \mathrm{V}^{-1} \mathrm{~m}^{-110}$. Although in general the underlying mechanism of the giant $\beta$ values remains unresolved, the internal E-field caused by charges trapped by defects in $\mathrm{MgO}$ can play an essential role ${ }^{9,11}$.

\footnotetext{
${ }^{1}$ Department of Physics and Astronomy, California State University Northridge, Northridge, California 91330, USA. ${ }^{2}$ Department of Electrical Engineering, University of California, Los Angeles, California 90095, USA. ${ }^{2}$ Inston Inc., Los Angeles, California 90095, USA. 'Present address: Physical and Computational Sciences Directorate, Pacific Northwest National Laboratory, Richland, Washington 99354, USA. Correspondence and requests for materials should be addressed to P.V.O. (email: phuong-vu.ong@pnnl.gov) or N.K. (email: nick.kioussis@csun.edu)
} 


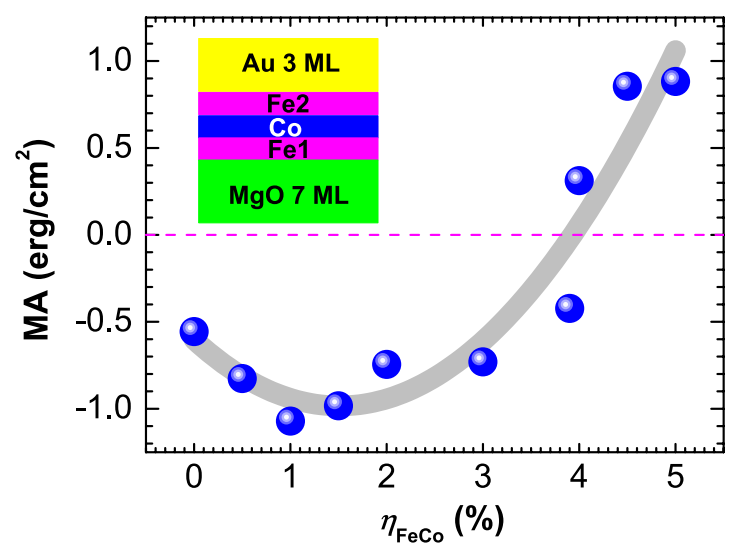

Figure 1. Strain dependence of zero-field MA where closed circles denote the ab initio results and the curve is a fit to equation (3).

Similarly the VCMA of Au/FM/I trilayers with different FM materials remains unresolved and controversial. A linear VCMA was reported in $\mathrm{Au} / \mathrm{Fe}_{80} \mathrm{Co}_{20} / \mathrm{MgO}$ junctions with $\beta=-38 \mathrm{fJ} \mathrm{V}^{-1} \mathrm{~m}^{-112}$. Interestingly, Shiota et al. observed a voltage-induced magnetization switching from in- to out-of-plane direction ${ }^{1}$. In contrast, $\mathrm{Au} / \mathrm{Fe} / \mathrm{MgO}$ exhibits a $\vee$-shape $\mathrm{VCMA}^{3}$. On the theoretical side, ab initio electronic structure calculations of Fe/ $\mathrm{MgO}^{13}$ and $\mathrm{Au} / \mathrm{Fe} / \mathrm{MgO}^{14}$ junctions with in-plane lattice constants of $\mathrm{Fe}$ and $\mathrm{MgO}$, respectively, found a linear VCMA with $\beta$ of about +130 and $+70 \mathrm{fJ} \mathrm{V}^{-1} \mathrm{~m}^{-1}$, respectively.

A ubiquitous feature in many $\mathrm{HM} / \mathrm{FM} / \mathrm{I}$ heterostructures is the large lateral strain among the HM, FM, and I components which can in turn tune the SOC and hence modify the magnetic anisotropy (MA) and magnetoelectric interfacial coupling. For example, nanostructures of $\mathrm{AuCu} / \mathrm{FeCo}$ core-shell has been shown to possess superior strain-induced magnetic properties such as high saturation magnetization and coercivity ${ }^{15}$.

HM cap can considerably modify MA of magnetic layers ${ }^{16}$ and a combined strain and capping effect can strongly enhance VCMA of a HM/FM/I junction ${ }^{17}$. However, a direct E-field induced switching of magnetization is still elusive in both computation and experiments $3,7-10,13,14,17-19$. This raises further questions that (i) If the synergistic effect of strain and HM on VCMA is robust for cap materials across the $5 d$ transition metals and (ii) Whether there exists a choice of HM which enables a direct E-field induced switching in a magnetic junction.

In this work we present $a b$ initio electronic structure calculations which demonstrate that epitaxial strain has a strong effect on $\mathrm{MA}$ of $\mathrm{Au} / \mathrm{FeCo} / \mathrm{MgO}$ trilayer in nonlinear manner, leading to a strain-induced spin-reorientation. Furthermore, strain gives rise to a rich VCMA behavior ranging from a $V$-shape to inverse- $V$-shape $(\wedge)$ E-field dependence with giant coefficient. This demonstrates the universal and robust VCMA behavior in strained HM/FM/I junction for HM across the $5 d$ transition metals. We also predict that an E-field-driven magnetization switching can be archived at low voltage.

Effect of strain on zero-field MA. Figure 1 shows the variation of the zero-field MA of the $\mathrm{Au} / \mathrm{FeCo} / \mathrm{MgO}$ junction with strain, $\eta_{\mathrm{FeC}}$. The inset shows the schematic structure of $\mathrm{Au} / \mathrm{FeCo} / \mathrm{MgO}$ trilayer. The iron atoms at the $\mathrm{Fe} / \mathrm{MgO}$ and $\mathrm{Fe} / \mathrm{Au}$ interfaces are denoted by $\mathrm{Fe} 1$ and $\mathrm{Fe} 2$, respectively. The system shows a nonlinear magnetoelastic (MEL) behavior with a spin-reorientation at $\sim 4 \%$ strain, in contrast to that in $\mathrm{Ta} / \mathrm{FeCo} / \mathrm{MgO}$ where MA is linearly dependent on strain with a magnetization switching occurs at $\sim 2.5 \%{ }^{17}$. This is a striking example on effect of HM cap on functional properties of a magnetic junction at nanoscale.

To explore the origin of the nonlinearity, we write the general expression MA $=\left[f_{M}\left(e_{n}, \alpha_{1}=1\right)-f_{M}\left(e_{n}, \alpha_{3}=1\right)\right] t$, where $t$ is the FM thickness. The total magnetic energy-density functional of the FM, $f_{M}$, is defined as

$$
f_{M}\left(e_{n}, \alpha_{k}\right)=\frac{K_{2}^{i}}{t}\left(1-\alpha_{3}^{2}\right)+f_{M E L}\left(e_{n}, \alpha_{k}\right),
$$

where $e_{n}(n=1-6)$ is strain tensor in Voigt notation, $\alpha_{k}(k=1-3)$ is direction cosine of the magnetization vector, and $K_{2}^{i}$ is the interface magnetocrystalline anisotropy. In a thin film grown epitaxially along the [001] crystallographic direction, there is no shear strain, i.e., $e_{5}=e_{5}=e_{6}=0$. The MEL energy density $f_{M E L}$ is expanded to second order $^{20}$ :

$$
\begin{aligned}
f_{M E L}\left(e_{n}, \alpha_{k}\right)= & B_{1} \sum_{k=1}^{3} e_{k} \alpha_{k}^{2}+\frac{1}{2} D_{11} \sum_{k=1}^{3} e_{k}^{2} \alpha_{k}^{2} \\
& +\frac{1}{2} D_{12} \sum_{\substack{j, k=1 \\
j \neq k}}^{3} e_{j} e_{k} \alpha_{j} \alpha_{k}
\end{aligned}
$$




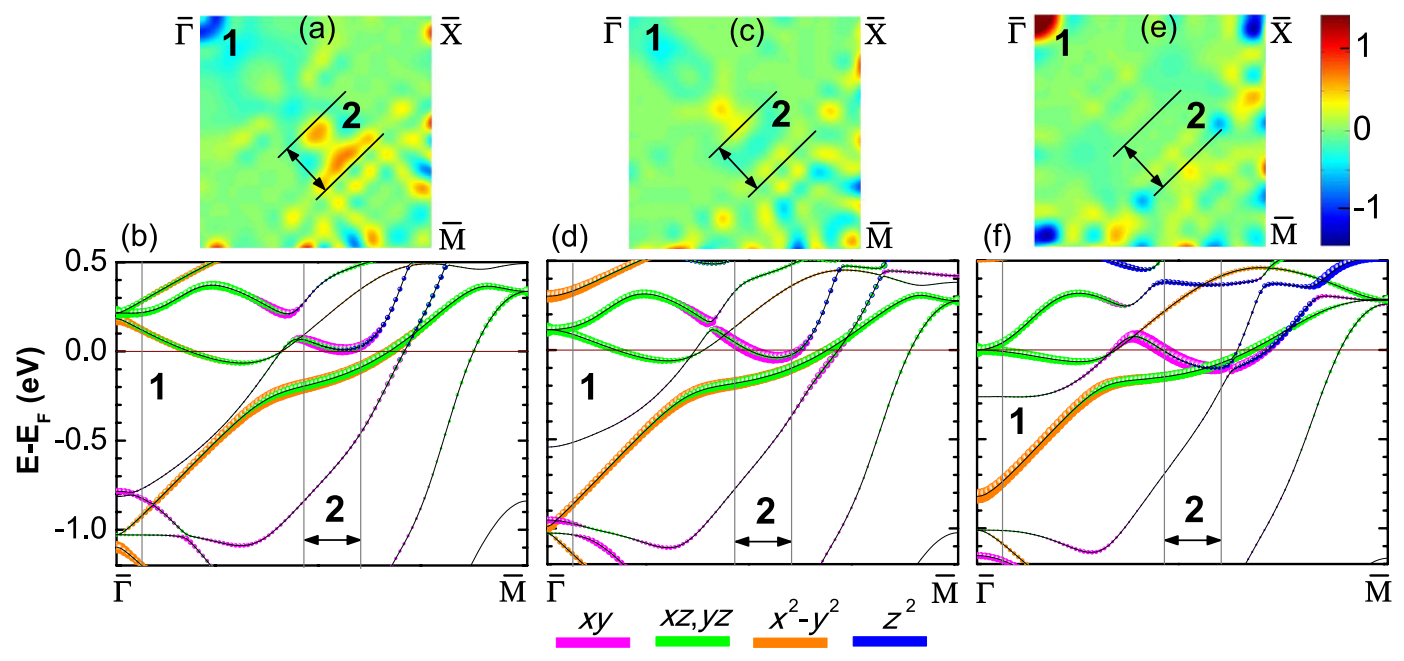

Figure 2. (a,c,e) $k$-resolved $\mathrm{MA}(\mathbf{k})$ (in $\mathrm{meV}$ ) in the $2 \mathrm{D} \mathrm{BZ}$ for $\eta_{F e C o}=0,2$ and $4 \%$, respectively. (b,d,f) Energyand $k$-resolved distributions of the orbital character of minority-spin bands along $\overline{\Gamma M}$ for the interfacial Fel atom $d$-states for $\eta_{\mathrm{FeCo}}=0,2$ and $4 \%$, respectively. Numerals in panels $(\mathbf{a}-\mathbf{f})$ refer to BZ $\mathbf{k}_{\|}$points $(\mathrm{BZPn}, \mathrm{n}=1,2)$ where there are large changes of MA under strain.

where $e_{1}=e_{2}=\eta_{\mathrm{FeCo}}$. The perpendicular strain $e_{3}$ is then determined by minimizing the energy density $f=f_{M}+f_{E L}$, where the elastic energy density $f_{E L}=\frac{1}{2} C_{11}\left(e_{1}^{2}+e_{2}^{2}+e_{3}^{2}\right)+C_{12}\left(e_{1} e_{2}+e_{2} e_{3}+e_{3} e_{1}\right)$, where $C_{i j}$ is the elastic stiffness tensor with $C_{11} \approx 2 C_{12}$ for transition metals ${ }^{21}$. The minimization gives rise to an MEL equation:

$$
\begin{aligned}
\mathrm{MA}= & K_{2}^{i}+\gamma\left(B_{1}^{2} / C_{11}\right)\left(2+D_{11} / C_{11}\right) t \\
& +(1+\gamma) B_{1} t \eta_{F e C o}+(1-\gamma)\left(D_{11} / 2\right) t \eta_{F e C o}^{2}
\end{aligned}
$$

where $\gamma=C_{11}^{2} /\left(C_{11}+D_{11}\right)^{2}$.

For $D_{11}=0$ or $\gamma=1$, the nonlinear term in equation (3) identically vanishes. A finite $D_{11}$ indicates that the presence of magnetization along a given direction induces an additional spontaneous strain on the FM film. Therefore, the combination of phenomenological analysis and ab initio calculation clearly shows that magnetic contribution to elastic behavior is significant and is the origin leading to the nonlinearity in the $\mathrm{Au} / \mathrm{FeCo} / \mathrm{MgO}$ trilayer. By fitting the $a b$ initio results we find $K_{2}^{i}=-0.61 \mathrm{erg} / \mathrm{cm}^{2}, B_{1}=-8.0 \times 10^{8} \mathrm{erg} / \mathrm{cm}^{3}$ and $D_{11}=44.8 \times 10^{10} \mathrm{erg} / \mathrm{cm}^{3}$. For bulk FeCo, the first-order MEL coefficient is $B_{1}^{v}=-3 \times 10^{8} \mathrm{erg} / \mathrm{cm}^{322}$. From the expression $B_{1}=B_{1}^{v}+B_{1}^{i} / t$, the interfacial contribution $B_{1}^{i}=-13.4 \mathrm{erg} / \mathrm{cm}^{2}$ can be inferred. For 100 -nm-thick Fe film on MgO experiments reported $B_{1}=-0.32 \times 10^{8} \mathrm{erg} / \mathrm{cm}^{3}$ and $D_{11}=1.1 \times 10^{10} \mathrm{erg} / \mathrm{cm}^{3}$. The value of $B_{1}$ is close to that of bulk Fe of $-0.34 \times 10^{8} \mathrm{erg} / \mathrm{cm}^{3}$ since the surface and interface effects are small in a thick Fe film ${ }^{23}$. Our values for $B_{1}$ and $D_{11}$ are an order of magnitude larger than these values and are consistent with the fact that $B_{1}^{v}$ of bulk $\mathrm{Fe}_{50} \mathrm{Co}_{50}$ is an order of magnitude larger than bulk Fe. Moreover, in the $\mathrm{Au} / \mathrm{FeCo} / \mathrm{MgO}$ nano-juction interface effects are crucial.

To elucidate the microscopic mechanism of the strain effect on MA, we calculate $k$-resolved MA according to the force theorem ${ }^{24}: \mathrm{MA}(\mathbf{k}) \approx \sum_{n \in o c c}\left[\varepsilon(n, \mathbf{k})^{[100]}-\varepsilon(n, \mathbf{k})^{[001]}\right]$ in the two-dimensional Brillouin zone (2D BZ). Here, $\varepsilon(n, \mathbf{k})^{[100]([001])}$ are the eigenvalues of the Hamiltonian for magnetization along the [100] ([001]) direction. Figure 2(a,c,e) display MA(k) for $\eta_{\mathrm{FeCo}}=0,2$ and 4\%, respectively. Figure 2(b,d,f) show the corresponding energyand $k$-resolved distribution of the orbital character of the minority-spin bands of the Fe1-derived $d_{x y}, d_{x z, y z}, d_{x^{2}-y^{2}}$, and $d_{z^{2}}$ states along $\overline{\Gamma M}$ for $\eta_{F e C o}=0,2$, and $4 \%$, respectively. We find that the strain-induced modification of the zero-field MA is mainly due to changes of the band structure of the interfacial Fel atom.

The MA can be expressed approximately in terms of the in- and out-of plane components of the orbital angular momentum operators $\hat{L}_{x(z)}$ as ${ }^{25}$

$$
\begin{aligned}
M C A \propto & \xi^{2} \sum_{o, u} \frac{\left|\left\langle\Psi_{o}^{\downarrow}\left|\hat{L}_{z}\right| \Psi_{u}^{\downarrow}\right\rangle\right|^{2}-\left|\left\langle\Psi_{o}^{\downarrow}\left|\hat{L}_{x}\right| \Psi_{u}^{\downarrow}\right\rangle\right|^{2}}{E_{u}^{\downarrow}-E_{o}^{\downarrow}} \\
& +\xi^{2} \sum_{o, u} \frac{\left|\left\langle\Psi_{o}^{\uparrow}\left|\hat{L}_{x}\right| \Psi_{u}^{\downarrow}\right\rangle\right|^{2}-\left|\left\langle\Psi_{o}^{\uparrow}\left|\hat{L}_{z}\right| \Psi_{u}^{\downarrow}\right\rangle\right|^{2}}{E_{u}^{\downarrow}-E_{o}^{\uparrow}} .
\end{aligned}
$$

Here, $\Psi_{o}^{\downarrow}\left(E_{o}^{\downarrow}\right), \Psi_{o}^{\uparrow}\left(E_{o}^{\uparrow}\right)$, and $\Psi_{u}^{\downarrow}\left(E_{u}^{\downarrow}\right)$ are occupied minority, occupied majority, and unoccupied minority-spin states (energies), and $\xi$ is the SOC constant. Unless otherwise stated throughout the remainder of this manuscript the $d$-states refer to minority-spin states. 

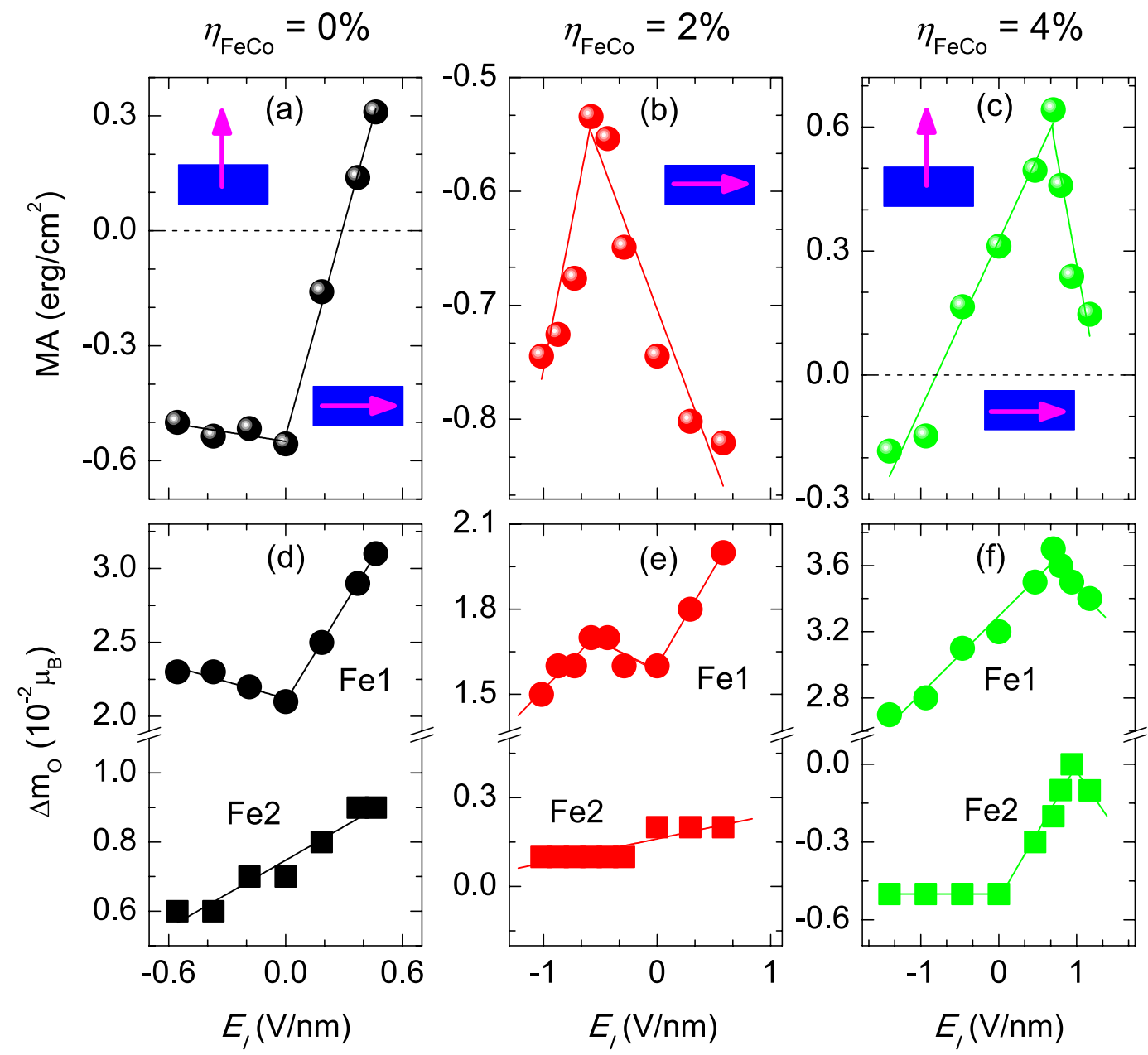

Figure 3. (a-c) MA versus E-field in $\mathrm{MgO}$ for different strain values. The vertical (horizonal) arrows indicate perpendicular (in-plane) magnetization. (d-f) Orbital moment difference, $\Delta m_{o}=m_{o}{ }^{[001]}-m_{o}{ }^{[100]}$, of the Fe1 and $\mathrm{Fe} 2$ interfacial atoms versus E-field for the same strain values.

At zero strain, there is a minimum and a maximum of $\mathrm{MA}\left(\mathbf{k}_{\|}\right)$in Fig. $2(\mathrm{a})$ around $\bar{\Gamma}(\mathrm{BZP} 1)$ and $\frac{1}{2} \overline{\Gamma M}$ (BZP2), respectively. The negative MA at BZP1 around $\bar{\Gamma}$ is due to the SOC of the minority-spin interfacial Fe1-derived occupied $d_{x y}$ and $d_{x^{2}-y^{2}}$ with the unoccupied $d_{x z, y z}$, through the $\hat{L}_{x}$ operator. On the other hand, at BZP2, there is strong SOC of the occupied Fe1 $d_{x^{2}-y^{2-}}$ and $d_{x z(y z)}$-derived states close to the Fermi energy with the unoccupied $d_{x y}$ and $d_{y z(x z)}$ through the out-of-plane $\hat{L}_{z}$, respectively. This in turn gives rise to a positive contribution to $\operatorname{MA}\left(\mathbf{k}_{\|}\right)$, which, however, is partially compensated by the negative contribution from SOC of the occupied Fe1 $d_{x z, y z}$ to the unoccupied $d_{x y}$ and $d_{z^{2}}$ derived states [Fig. 2(b)].

At $\eta_{\mathrm{FeCo}}=2 \%$ [Fig. 2(c,d)] there is a large downward shift of the occupied Fe $1 d_{x y}$ around $\bar{\Gamma}$ leading to an increase of its energy separation to the unoccupied Fe $1 d_{x z, y z}$ states and hence an increase of MA(BZP1). At BZP2, the unoccupied $d_{x y}$ and $d_{x z, y z}$ slightly above the Fermi energy shift down and become occupied. This eliminates in turn the SOC of the occupied Fel $d_{x^{2}-y^{2-}}$ and $d_{x z(y z)}$-derived states with the unoccupied $d_{x y}$ and $d_{y z(x z)}$ through $\hat{L}_{z}$, respectively. Consequently, the MA(BZP2) is reduced. Because the $\mathrm{MA}\left(\mathbf{k}_{\|}\right)$increases at BZP1 and decreases at $\mathrm{BZP} 2$, the total MA hardly changes upon increasing the strain from 0 to $2 \%$ (Fig. 1).

Under 4\% strain the $k$-resolved MA, shown in Fig. 2(e), exhibits a sharp positive peak at BZP1 which is responsible for the magnetization vector switching from an in- to out-of-plane direction. The underlying origin in the electronic structure is that one of the Fe $1 d_{x z(y z)}$ states at BZP1 slightly above the Fermi energy shifts downward and becomes occupied [Fig. 2(f)]. This in turn induces SOC of the occupied Fe1 $d_{x z(y z)}$ with the unoccupied Fe1 $d_{y z(x z)}$ around BZP1 through the out-of-plane orbital angular momentum operator $\hat{L}_{z}$.

Effect of strain on VCMA. The variation of MA as a function of the E-field in MgO is shown in Fig. 3(a-c) for $\eta_{\mathrm{FeCo}}=0,2$ and $4 \%$, respectively. The E-field in the insulator is inversely proportional to the strain-dependent out-of-plane component, $\varepsilon_{\perp}$, of the dielectric tensor of the insulator. We find that $\varepsilon_{\perp}$ increases exponentially with increasing compressive strain on the insulator (i.e., decreasing expansive strain on the FM). The calculated values of the relative $\varepsilon_{\perp} / \varepsilon_{0}$ are $10.7,17.0$, and 27.0 for for $\eta_{F e C o}=4,2$, and $0 \%$, respectively. 

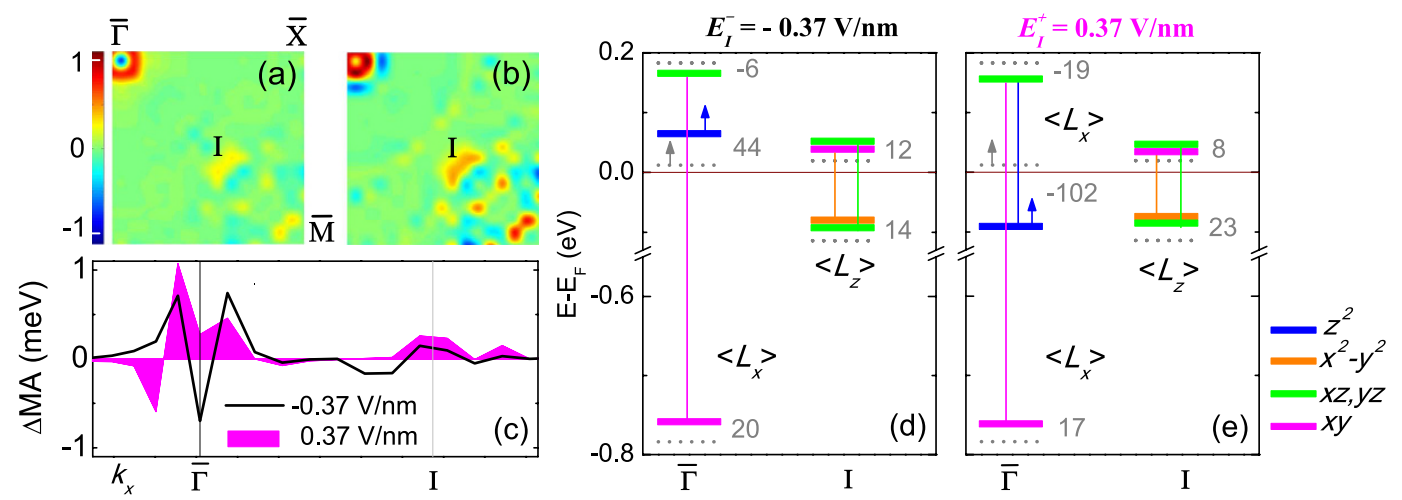

Figure 4. Zero strain: (a,b) E-field-induced change of $\mathrm{MA}, \triangle \mathrm{MA}(\mathbf{k})$, (in $\mathrm{meV})$ in $2 \mathrm{D} \mathrm{BZ}$ for $E_{I}^{-}=-0.37 \mathrm{~V} / \mathrm{nm}$ and $E_{I}^{+}=0.37 \mathrm{~V} / \mathrm{nm}$, respectively. (c) The E-field-induced $\triangle \mathrm{MA}(\mathbf{k})$ along symmetry directions. (d,e) Fe1derived electronic levels at the $\bar{\Gamma}$ and I points (solid horizontal lines) under the negative and positive E-fields, where the numerals indicate shifts (in $\mathrm{meV}$ ) of the energy levels compared to those at zero field (dotted lines). Majority-spin states are indicated by upward arrows and vertical lines connecting pairs of occupied and unoccupied states denote nonvanishing SOC matrix-elements where the line color matches that of the occupied state.

The results in Fig. 3(a-c) demonstrate that epitaxial strain gives rise to a wide range of intriguing VCMA behavior where the MA changes from (i) asymmetric $\vee$-shape field behavior under $0 \%$ strain with $\beta$ values of $1871(-101) \mathrm{fJ} \mathrm{V}^{-1} \mathrm{~m}^{-1}$ for positive (negative) E-field; to (ii) asymmetric $\wedge$-shape under $2 \%$ strain with $\beta$ values of $-246(482) \mathrm{fJ} \mathrm{V} \mathrm{V}^{-1} \mathrm{~m}^{-1}$ for E-field larger (smaller) than the critical field $E_{c}=-0.58 \mathrm{~V} / \mathrm{nm}$ where the MA reaches its maximum; and to (iii) asymmetric $\wedge$-shape under $4 \%$ strain with $\beta$ values of -1061 (393) $\mathrm{fJ} \mathrm{V}^{-1} \mathrm{~m}^{-1}$ for $E \geq(\leq)$ $E_{c}=0.70 \mathrm{~V} / \mathrm{nm}$. Note that the range of $E_{I}$ is below the breakdown field of $\mathrm{MgO}(\sim 1 \mathrm{~V} / \mathrm{nm})$. In most experiments $E_{I}$ is below $0.7 \mathrm{~V} / \mathrm{nm}^{4,7}$, which is the value of $E_{c}$ at $4 \%$. Therefore, experimentally the VCMA appears linear at $4 \%$. These VCMA coefficient values are the highest reported today and are larger by one to two order of magnitude compared to those reported in most experiments, except for those in refs 8,9 where charged defects may play a role.

More importantly, we predict an E-field-driven switching of the magnetic easy axis from in-plane to out-of-plane direction at $0.30(-0.80) \mathrm{V} / \mathrm{nm}$ for $\eta_{\mathrm{FeCo}}=0(4) \%$. These findings have two important implications for magnetoelectric spintronics. First, the predicted VCMA coefficient values are very close to or larger than the critical value of $\sim 200 \mathrm{fJ} \mathrm{V}^{-1} \mathrm{~m}^{-1}$ required to achieve a switching bit energy below $1 \mathrm{fJ}$ in the next-generation of MeRAMs. Second, the results reveal the feasibility of tailoring the VCMA behavior via strain engineering to achieve desired MeRAM devices.

Figure $3(\mathrm{~d}-\mathrm{f})$ show the difference between the out-of- and in-plane orbital moments, $\Delta m_{o}=m_{o}{ }^{[001]}-m_{o}{ }^{[100]}$, of the Fe1 and $\mathrm{Fe} 2$ interfacial atoms as a function of E-field for $\eta_{\mathrm{FeCo}}=0,2$ and $4 \%$, respectively. The E-field variation of $\Delta m_{o}$ for $\mathrm{Co}$ is much weaker and is not shown here. For single atomic species FMs with large exchange splitting the MA is related to the orbital magnetic moment anisotropy via the Bruno expression $\mathrm{MA}=\xi \Delta m_{o} /\left(4 \mu_{B}\right)^{26}$. However, for structures consisting of multiple atomic species (as in the case of trilayers) with strong hybridization it has been shown that the expression is not satisfied and needs to be modified ${ }^{27}$. Overall the E-field dependence of $\Delta m_{o}$ for Fel and to a lesser degree of Fe 2 correlates with that of the MA.

From the equation (4), E-field induced MA can originate from one or both of the following mechanisms: (i) charge screening or band filling effect, which is described by changes of the numerators and (ii) changes in separation of spin-orbit coupled pairs, which are described by the denominators. Thus far most of works assume either the band filling effect ${ }^{13,28-30}$ or the change in energy separation ${ }^{14,31}$ to be the predominant contribution. In the present work, we do not exclude possible contributions from the band filling effect. However, from our analysis the E-field induced changes in band structures can provide a consistent explanation of the E-field induced MA behavior, indicating that this is a plausible mechanism.

VCMA at zero strain. In order to understand the VCMA behavior under zero strain we show in Fig. 4(a,b) the E-field induced change of MA, $\Delta \mathrm{MA}(\mathbf{k})=\mathrm{MA}(\mathbf{k}, E)-\mathrm{MA}\left(\mathbf{k}, E_{c}=0\right)$, in the $2 \mathrm{D} \mathrm{BZ}$ for $E_{I}^{-}=-0.37 \mathrm{~V} / \mathrm{nm}$ and $E_{I}^{+}=0.37 \mathrm{~V} / \mathrm{nm}$, respectively. Integration of the $\triangle \mathrm{MA}(\mathbf{k})$ over the $2 \mathrm{D} \mathrm{BZ}$ for negative and positive fields yields induced MA consistent with the asymmetric $\vee$-shape VCMA in Fig. 3(a). We also show in Fig. 4(c) the E-field induced $\triangle \mathrm{MA}(\mathbf{k})$ along symmetry lines for positive and negative E-fields. Figure 4(d,e) display the E-field induced shifts of the energy levels (horizontal lines) of the Fe1-derived $d$ states and the non-vanishing SOC matrix elements (vertical lines) between occupied and unoccupied $d$ states at the $\bar{\Gamma}$ and I BZ points, where there are large changes of the MA.

The decrease of MA at $\bar{\Gamma}$ under negative E-field is due to the fact that the occupied minority-spin Fe $1 d_{x y}$ shifts upward while the unoccupied minority-spin Fel $d_{x z, y z}$ states shift downward. Consequently the energy separation of this pair of states, coupled via the in-plane orbital angular momentum, $\hat{L}_{x}$, decreases resulting in a $\Delta \mathrm{MA}(\bar{\Gamma})<0$. On the other hand, the increase of MA at $\bar{\Gamma}$ under positive E-field is associated with a large down- 

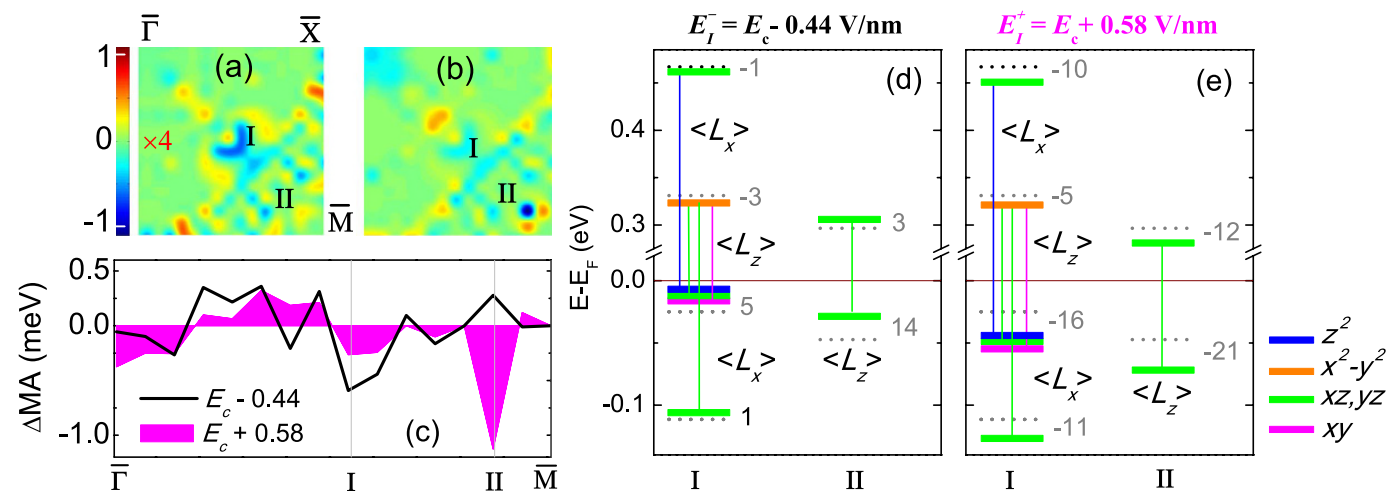

Figure 5. The same as Fig. $4(\mathrm{a}-\mathrm{e})$ but for $2 \%$ strain and $E_{I}^{-}=E_{c}-0.44 \mathrm{~V} / \mathrm{nm}$ and $E_{I}^{+}=E_{c}+0.58 \mathrm{~V} / \mathrm{nm}$. The level shifts are with respect to those at the critical field $E_{c}=-0.58 \mathrm{~V} / \mathrm{nm}$. For the sake of clarity, the contour values in (a) are magnified by 4 times.

ward shift of the unoccupied majority-spin Fe1 $d_{z^{2}}$ state to $\sim 0.1 \mathrm{eV}$ below the Fermi energy (Fig. 4(e)). This in turn induces a spin-mixed SOC between the majority-spin state and the unoccupied minority-spin $d_{x z y z}$ states via the in-plane orbital angular momentun $\hat{L}_{x}$ [second term in equation (4)], rendering $\triangle \mathrm{MA}(\bar{\Gamma})>0$.

At BZ point I under both negative and positive E-field the Fe1 minority-spin occupied $d_{x z, y z}$ and $d_{x^{2}-y^{2}}$ and unoccupied $d_{x z, y z}$ and $d_{x y}$ states shift upward resulting in a decrease of the energy separation between the occupied-unoccupied pairs by $2 \mathrm{meV}$ and $15 \mathrm{meV}$, respectively. This leads to an increase of the MA contribution of the $\hat{L}_{z}$ SOC matrix elements between (i) the occupied $d_{x z(y z)}$ and unoccupied $d_{y z(x z)}$ states and (ii) the occupied $d_{x^{2}-y^{2}}$ and unoccupied $d_{x y}$ states, rendering $\Delta \mathrm{MA}(\mathbf{k})>0$. The larger increase of $\Delta \mathrm{MA}(\mathbf{k})$ at $\mathrm{BZ}$ point I under positive field correlates with the larger decrease of energy separation of the SOC pairs under positive E-field compared to that for negative E-field.

VCMA at $2 \%$ strain. Figure $5(\mathrm{a}, \mathrm{b})$ show the E-field induced change of $\mathbf{k}$-resolved MA, $\triangle \mathrm{MA}(\mathbf{k})=\mathrm{MA}(\mathbf{k}, E)$ $\operatorname{MA}\left(\mathbf{k}, E_{c}\right)$, in the $2 \mathrm{D} \mathrm{BZ}$ for $E_{I}^{-}=E_{c}-0.44 \mathrm{~V} / \mathrm{nm}$ and $E_{I}^{+}=E_{c}+0.58 \mathrm{~V} / \mathrm{nm}$, where $E_{c}=-0.58 \mathrm{~V} / \mathrm{nm}$ is the critical E-field at $2 \%$ strain [Fig. 3(b)]. The contour values in Fig. 5(a) are magnified 4 times for the sake of clarity. Integration of the $\triangle \mathrm{MA}(\mathbf{k})$ over the $2 \mathrm{D} \mathrm{BZ}$ for $E_{I}^{-}$and $E_{I}^{+}$E-field yields induced MA consistent with the asymmetric $\vee$-shape VCMA in Fig. 3(b). The $\triangle M A(k)$ is plotted along symmetry lines in Fig. 5(c). In Fig. 5(d,e) we show the E-field induced shifts of the energy levels of the minority-spin Fe1-derived $d$ states with respect to those at the critical field and the non-vanishing SOC matrix elements at the $\bar{\Gamma}$, I and II BZ points, where there are significant changes of the MA.

Under $E_{I}^{-}$, at the I BZ point the degenerate occupied Fel $d_{x y}, d_{x z, y z}$, and $d_{z^{2}}$ states near the Fermi level shift up while the unoccupied $d_{x^{2}-y^{2}}$ shifts down. This leads to an increase in the positive MA contribution of the $\hat{L}_{z}$ SOC matrix elements between the occupied Fel $d_{x y}$ and unoccupied $d_{x^{2}-y^{2}}$ states. On the other hand, the E-field induced energy shifts decrease further the negative MA contribution of $\hat{L}_{x}$ between (i) occupied Fe1 $d_{x z, y z}$ with unoccupied $d_{x^{2}-y^{2}}$ states and (ii) occupied $d_{z^{2}}$ with the unoccupied $d_{x z, y z}$ states. The interplay between the in- and out-of-plane orbital angular momentum matrix elements results in a net negative $\triangle \mathrm{MA}(\mathbf{k})$. In sharp contrast the $E_{I}^{+}$increases the separation between the occupied Fel $d_{x y}$ and unoccupied $d_{x^{2}-y^{2}}$ minority-spin states which are coupled through $\hat{L}_{z}$. This in turn decreases the positive MA contribution of the SOC between these states rendering $\triangle \mathrm{MA}(\mathbf{k})<0$.

At BZ point II $\triangle \mathrm{MA}(\mathbf{k})$ is positive (negative) for $E_{I}^{-}\left(E_{I}^{+}\right)$[Fig. 5(c)]. This is due to the decrease (increase) of the energy separation between the occupied Fel $d_{x z(y z)}$ - and unoccupied $d_{y z(x z)}$-derived states, coupled through $\hat{L}_{z}$, under $E_{I}^{-}\left(E_{I}^{+}\right)$[Fig. 5(d,e)].

In summary, we have demonstrated that epitaxial strain, which is ubiquitous in many HM/FM/I trilayers, has a dramatic effect on the VCMA. It can change the VCMA from a $\vee$ - to a $\wedge$-shape E-field dependence with giant VCMA coefficients and tunable critical E-field. Furthermore, we have predicted that tuning of epitaxial strain can give rise to an E-field induced magnetization switching at low voltage. These result demonstrate that the universality and robustness of the VCMA behavior in strained HM/FM/I trilayers and that efficient E-field-driven magnetic switching can be attained by design. These findings open interesting prospects for exploiting strain engineering to harvest higher efficiency VCMA for the next generation MeRAM devices.

\section{Methods}

The $a b$ initio calculations have been carried out within the framework of the projector augmented-wave formal$\mathrm{ism}^{32}$, as implemented in the Vienna ab initio simulation package (VASP) ${ }^{33,34}$. The generalized gradient approximation $^{35}$ was employed to treat the exchange-correlation potential. To simulate the epitaxial growth of the $\mathrm{Au} / \mathrm{FeCo} / \mathrm{MgO}$ trilayer we employed a slab supercell along [001] consisting of three monolayers (MLs) of fcc $\mathrm{Au}$, three MLs of B2-type FeCo, seven MLs of rock-salt $\mathrm{MgO}$ and a $15 \AA$-thick vacuum region separating the periodic slabs. The $\langle 110\rangle$ axis of $\mathrm{MgO}$ and $\mathrm{Au}$ are aligned with the $\langle 100\rangle$ axis of $\mathrm{FeCo}$ and the $\mathrm{O}$ atoms at the 
$\mathrm{FeCo} / \mathrm{MgO}$ interface are placed atop of $\mathrm{Fe}$ atoms. The iron atoms at the $\mathrm{Fe} / \mathrm{MgO}$ and $\mathrm{Fe} / \mathrm{Au}$ interfaces are denoted by $\mathrm{Fe} 1$ and $\mathrm{Fe} 2$, respectively [Fig. 1 inset]. Due to the large lattice constant mismatch between $\mathrm{MgO}$ and FeCo, the $\mathrm{FeCo}(\mathrm{MgO})$ is under expansive (compressive) strain, $\eta_{F e C o}\left(\eta_{M g O}\right)$, of $\sim+4 \%(-5.6 \%)$ compared with the lattice of bulk FeCo (MgO). Depending on the experimental conditions $\eta_{\mathrm{FeCo}}$ can vary from zero to $4 \%^{36}$. At each strain, the magnetic and electronic degrees of freedom and atomic $z$ positions are relaxed in the presence of the E-field until the forces acting on the ions become less than $\times 10^{-3} \mathrm{eV} / \AA$ and the change in the total energy between two ionic relaxation steps is smaller than $10^{-6} \mathrm{eV}$. The plane-wave cutoff energy was set to $500 \mathrm{eV}$ and a $15 \times 15 \times 1$ Monkhorst-Pack $k$-mesh was used for the relaxation calculations. The SOC of the valence electrons is in turn included using the second-variation method ${ }^{37}$ employing the scalar-relativistic eigenfunctions of the valence states and a $31 \times 31 \times 1 \mathrm{k}$-point mesh. The MA per unit interfacial area, $\mathrm{A}$, is determined from $\mathrm{MA}=\left[E_{[100]}-E_{[001]}\right] / \mathrm{A}$, where $E_{[100]}$ and $E_{[001]}$ are the total energies with magnetization along the [100] and [001] directions, respectively.

\section{References}

1. Shiota, Y. et al. Voltage-assisted magnetization switching in ultrathin $\mathrm{Fe}_{80} \mathrm{Co}_{20}$ alloy layers. Appl. Phys. Express 2, 063001 (2009).

2. Shiota, Y. et al. Induction of coherent magnetization switching in a few atomic layers of FeCo using voltage pulses. Nature Mater. 11, 39-43 (2012).

3. Maruyama, T. et al. Large voltage-induced magnetic anisotropy change in a few atomic layers of iron. Nature Nanotech. 4, 158-161 (2009).

4. Wang, W.-G., Li, M., Hageman, S. \& Chien, C. L. Electric-field-assisted switching in magnetic tunnel junctions. Nature Mater. 11, 64-68 (2012).

5. Ikeda, S. et al. A perpendicular-anisotropy CoFeB/MgO magnetic tunnel junction. Nature Mater. 9, 721-724 (2010).

6. Wang, K. L., Alzate, J. G. \& Amiri, P. K. Low-power non-volatile spintronic memory: STT-RAM and beyond. J. Phys. D: Appl. Phys. 46, 074003 (2013).

7. Endo, M., Kanai, S., Ikeda, S., Matsukura, F. \& Ohno, H. Electric-field effects on thickness dependent magnetic anisotropy of sputtered $\mathrm{MgO} / \mathrm{Co}_{4} 0 \mathrm{Fe}_{4} 0 \mathrm{~B}_{2} 0 /$ Ta structures. Appl. Phys. Lett. 96, 212503 (2010).

8. Bonell, F. et al. Large change in perpendicular magnetic anisotropy induced by an electric field in FePd ultrathin films. Appl. Phys. Lett. 98, 232510 (2011).

9. Rajanikanth, A., Hauet, T., Montaigne, F., Mangin, S. \& Andrieu, S. Magnetic anisotropy modified by electric field in V/Fe/ $\mathrm{MgO}(001) / \mathrm{Fe}$ epitaxial magnetic tunnel junction. Appl. Phys. Lett. 103, 062402 (2013).

10. Nozaki, T. et al. Voltage-induced magnetic anisotropy changes in an ultrathin FeB Layer sandwiched between two MgO layers. Appl. Phys. Express 6, 073005 (2013).

11. Bauer, U., Przybylski, M., Kirschner, J. \& Beach, G. S. D. Magnetoelectric charge trap memory. Nano Letters 12, 1437-1442 (2012).

12. Nozaki, T., Shiota, Y., Shiraishi, M., Shinjo, T. \& Suzuki, Y. Voltage-induced perpendicular magnetic anisotropy change in magnetic tunnel junctions. Appl. Phys. Lett. 96, 022506 (2010).

13. Niranjan, M. K., Duan, C.-G., Jaswal, S. S. \& Tsymbal, E. Y. Electric field effect on magnetization at the Fe/MgO(001) interface. Appl. Phys. Lett. 96, 222504 (2010).

14. Nakamura, K., Akiyama, T., Ito, T., Weinert, M. \& Freeman, A. J. Role of an interfacial FeO layer in the electric-field-driven switching of magnetocrystalline anisotropy at the Fe/MgO interface. Phys. Rev. B 81, 220409 (2010).

15. Gong, M., Kirkeminde, A., Wuttig, M. \& Ren, S. Phase transformation-induced tetragonal FeCo nanostructures. Nano Letters 14, 6493-6498 (2014).

16. Ong, P. V. et al. Electric field control and effect of Pd capping on magnetocrystalline anisotropy in FePd thin films: A first-principles study. Phys. Rev. B 89, 094422 (2014).

17. Ong, P. V. et al. Giant voltage modulation of magnetic anisotropy in strained heavy metal/magnet/insulator heterostructures. Phys. Rev. B 92, 020407(R) (2015).

18. Li, X. et al. Thermally stable voltage-controlled perpendicular magnetic anisotropy in $\mathrm{Mo}|\mathrm{CoFeB}| \mathrm{MgO}$ structures. Appl. Phys. Lett. 107, 142403 (2015)

19. Yu G. et al. Strain-induced modulation of perpendicular magnetic anisotropy in $\mathrm{Ta} / \mathrm{CoFeB} / \mathrm{MgO}$ structures investigated by ferromagnetic resonance. Appl. Phys. Lett. 106, 072402 (2015); 106, 169902 (2015) (Erratum).

20. Eastman, D. E. Ultrasonic study of first-order and second-order magnetoelastic properties of yttrium iron garnet. Phys. Rev. 148, 530-542 (1966).

21. Sander, D. The correlation between mechanical stress and magnetic anisotropy in ultrathin films. Rep. Prog. Phys. 62, 809-858 (1999).

22. Clark, A. E., Restorff, J. B., WunFogle, M., Wu, D. \& Lograsso, T. A. Temperature dependence of the magnetostriction and magnetoelastic coupling in $\mathrm{Fe}_{100-x} \mathrm{Al}_{x}(x=14.1,16.6,21.5,26.3)$ and $\mathrm{Fe}_{50} \mathrm{Co}_{50}$. J. Appl. Phys. 103, 07B310 (2008).

23. Koch, R., Weber, M., Thürmer, K. \& Rieder, K. H. J. Magnetoelastic coupling of Fe at high stress investigated by means of epitaxial $\mathrm{Fe}(001)$ films. Magn. Magn. Mat. 159, L11-L16 (1996).

24. Weinert, M., Watson, R. E. \& Davenport, J. W. Total-energy differences and eigenvalue sums. Phys. Rev. B 32, 2115 (1985).

25. Wang, D.-S., Wu, R. \& Freeman, A. J. First-principles theory of surface magnetocrystalline anisotropy and the diatomic-pair model. Phys. Rev. B 47, 14932 (1993).

26. Weller, D. et al. Orbital magnetic moments of Co in multilayers with perpendicular magnetic anisotropy. Phys. Rev. B 49, 12888 (1994).

27. Andersson, C. et al. Influence of ligand states on the relationship between orbital moment and magnetocrystalline anisotropy. Phys. Rev. Lett. 99, 177207 (2007).

28. Ruiz-Daz, P., Dasa, T. R. \& Stepanyuk, V. S. Tuning magnetic anisotropy in metallic multilayers by surface charging: an $a b$ initio study. Phys. Rev. Lett. 110, 267203 (2013).

29. Tsujikawa, M. \& Oda, T. Finite electric field effects in the large perpendicular magnetic anisotropy surface $\mathrm{Pt} / \mathrm{Fe} / \mathrm{Pt}(001): \mathrm{a}$ firstprinciples study. Phys. Rev. Lett. 102, 247203 (2009).

30. Duan, C.-G. et al. Surface magnetoelectric effect in ferromagnetic metal films. Phys. Rev. Lett. 101, 137201 (2008).

31. Nakamura, K. et al. Giant modification of the magnetocrystalline anisotropy in transition-metal monolayers by an external electric field. Phys. Rev. Lett. 102, 187201 (2009).

32. Blöchl, P. E. Projector augmented-wave method. Phys. Rev. B 50, 17953 (1994).

33. Kresse, G. \& Furthmüller, J. Efficient iterative schemes for ab initio total-energy calculations using a plane-wave basis set. Phys. Rev. B 54, 11169 (1996).

34. Kresse, G. \& Furthmüller, J. Efficiency of ab-initio total energy calculations for metals and semiconductors using a plane-wave basis set Comput. Mater. Sci. 6, 15-50 (1996).

35. Perdew, J. P. et al. Atoms, molecules, solids, and surfaces: Applications of the generalized gradient approximation for exchange and correlation. Phys. Rev. B 46, 6671 (1992) 
36. Yuasa, S., Nagahama, T., Fukushima, A., Suzuki, Y. \& Ando, K. Giant room-temperature magnetoresistance in single-crystal $\mathrm{Fe} / \mathrm{MgO} / \mathrm{Fe}$ magnetic tunnel junctions. Nature Mater. 3, 868-871 (2004).

37. Koelling, D. D. \& Harmon, B. N. A technique for relativistic spin-polarised calculations. J. Phys C: Solid State Phys. 10, 3107 (1977).

\section{Acknowledgements}

This research was supported by NSF Grant No. ERC-Translational Applications of Nanoscale Multiferroic Systems (TANMS)-1160504 and in part by Inston Inc. through a Phase II NSF Small Business Innovation Research award.

\section{Author Contributions}

P.V.O. carried out calculations, prepared the Figures 1-5, and wrote the first draft of the manuscript. P.V.O. and N.K. revised the manuscript and discussed data. N.K. supervised the project. All the authors have analyzed and discussed the results thoroughly and contributed to the writing of the manuscript.

\section{Additional Information}

Competing financial interests: The authors declare no competing financial interests.

How to cite this article: Ong, P. V. et al. Electric-field-driven magnetization switching and nonlinear magnetoelasticity in Au/FeCo/MgO heterostructures. Sci. Rep. 6, 29815; doi: 10.1038/srep29815 (2016).

(c) (i) This work is licensed under a Creative Commons Attribution 4.0 International License. The images or other third party material in this article are included in the article's Creative Commons license, unless indicated otherwise in the credit line; if the material is not included under the Creative Commons license, users will need to obtain permission from the license holder to reproduce the material. To view a copy of this license, visit http://creativecommons.org/licenses/by/4.0/ 